\title{
Phaseless spherical near-field antenna measurements for low and medium gain antennas
}

\author{
C. H. Schmidt ${ }^{1,2}$, S. F. Razavi ${ }^{1}$, T. F. Eibert ${ }^{2}$, and Y. Rahmat-Samii ${ }^{1}$ \\ ${ }^{1}$ Department of Electrical Engineering, University of California, Los Angeles, USA \\ ${ }^{2}$ Lehrstuhl für Hochfrequenztechnik, Technische Universität München, Germany
}

\begin{abstract}
The characterisation of antenna radiation patterns by measurements in the near-field and a following near-field far-field transformation require accurate amplitude and phase data. Especially at higher frequencies phase measurements are demanding in terms of instrumentation and measurement accuracy. Phaseless techniques which require amplitude only data on one or more measurement surfaces are therefore of special interest. In this paper a phaseless spherical algorithm based on spherical modal expansion is presented. The algorithm works with amplitude only near-field data measured on two spheres of different radii and is therefore not restricted to certain types of antennas as for planar measurements for example. Simulated as well as measured results of low and medium gain antennas are shown.
\end{abstract}

\section{Introduction}

The radiation characteristic of an antenna under test (AUT) is typically defined at far-field distance and is therefore to be measured in the far-field region of the AUT. Two common alternatives to direct measurements in the far-field are compact range measurements (Balanis, 2008) which emulate far-field conditions at close distance to the AUT and secondly near-field measurement techniques. The far-field of an AUT consists of the same modes as the near-field. Therefore it is possible to determine performance of the AUT from a measurement in the radiating near-field. The field modes are determined and processed to the far-field by a post-processing near-field far-field transformation (Yaghjian, 1986). Especially for electrically large AUTs which satisfy far-field conditions at a far distance $\left(\approx 2 D^{2} / \lambda, D\right.$ being the diameter of the minimum sphere enclosing the AUT and $\lambda$ the wavelength), near-field measurements can be carried out much

Correspondence to: C. H. Schmidt (carstenschmidt@tum.de) closer to the AUT in relatively small sized measurement facilities.

A critical requirement for the near-field far-field transformation is the accurate knowledge of the near-fields amplitude and phase. Amplitude measurements are less critical in terms of measurement equipment and accuracy compared to phase measurements, even for high frequencies. This is why phaseless measurement techniques (Yaccarino and RahmatSamii, 1999; Pierri et al., 1999; Las-Heras and Sarkar, 2002; Costanzo et al., 2005; Soldovieri et al., 2005; Schejball et al., 2008) have obtained considerable interest in research. Costly vector network analysers are no longer required for phase measurements and also phaseless techniques show some superior robustness for some classes of errors (Razavi and Rahmat-Samii, 2008). The lack of phase information is often compensated either by further amplitude only measurements on additional surfaces or by measurements with additional probes. The Iterative Fourier Technique (IFT) (Razavi and Rahmat-Samii, 2006) is known from planar phaseless measurements and requires amplitude only measurements on two planes. Planar measurement techniques (Kerns, 1981) rely on the finite sized scan plane in front of the AUT and are therefore only suitable for highly directive antennas.

A more common approach for the measurement of medium and low gain antennas is the spherical measurement technique (Hansen, 1988), where the radiated fields of the AUT over a sphere are recorded and the radiation in backward direction is also considered. To utilise the advantages of phaseless measurements, a phaseless spherical transformation algorithm based on a spherical modal expansion has been presented (Schmidt and Rahmat-Samii, 2009) and applied to a low gain patch antenna. The algorithm is particularly suitable for low as well as medium gain antennas and utilises measured amplitude only near-field data on two spheres. A modal expansion representing the radiation characteristic of the AUT is obtained in an iterative manner with some similarities to the IFT.

In this paper the phaseless spherical algorithm is further studied, applied and extended to the needs of medium 


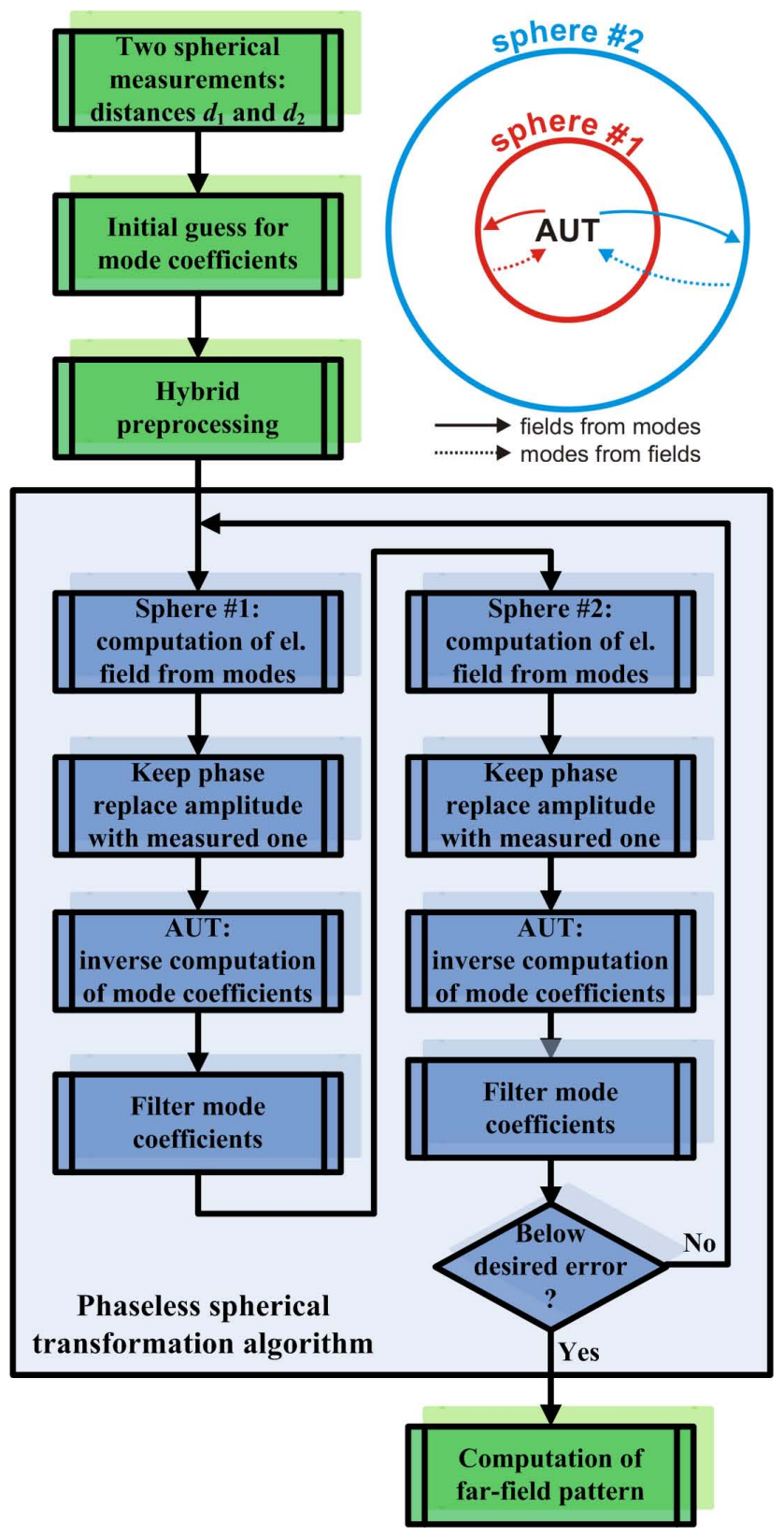

Fig. 1. Flowchart of phaseless spherical transformation algorithm working with amplitude only data on two spheres.

gain antenna measurements. In Sect. 2 a brief fundamental background of spherical near-field measurements is given whereas Sect. 3 introduces the phaseless spherical algorithm. Section 4 shows measured results of a low gain patch antenna and considers simulated near-field scenarios of a medium gain horn antenna. Characteristics of medium gain antennas within the phaseless spherical algorithm are addressed considering the example of a simulated Narda standard gain horn.

\section{Spherical near-field far-field transformation}

For spherical antenna measurements the radiated field distribution of an AUT

$\boldsymbol{E}(r, \varphi, \vartheta)=\frac{k}{\sqrt{\eta}} \sum_{s=1}^{2} \sum_{n=1}^{\infty} \sum_{m=-n}^{n} Q_{s m n} \boldsymbol{F}_{s m n}(r, \varphi, \vartheta)$

can be expanded in outgoing spherical modes $\boldsymbol{F}_{s m n}(r, \varphi, \vartheta)$ (Hansen, 1988). The radiation characteristic of the AUT is obtained by superimposing all considered modes weighted with the mode expansion coefficients $Q_{s m n}$ characterising the AUT. $k$ is the wavenumber and $\eta$ the free space wave admittance. For practical measurements the infinite number of modes required to characterise the AUT is truncated to

$N=\left\lfloor\pi \frac{D}{\lambda}\right\rfloor+10$

depending on the electrical size of the AUT $D / \lambda$ (Balanis, 2008).

$\{Q\}=[\mathbf{C}]^{-1} \cdot\{\boldsymbol{E}\}$

are obtained by inverse solution of the field problem. $[\mathbf{C}]$ is the coupling matrix, containing discrete values of the spherical modes for discrete measurement points. $\{\boldsymbol{E}\}$ is the vector containing the amplitude and phase near-field values measured on a sphere. Once the mode expansion coefficients are known, the desired far-field pattern can be computed using asymptotic expressions for the spherical modes (Hansen, 1988).

In general a probe correction is required to compensate for the field probe effects on a near-field measurement. For spherical measurements and in particular if the probe is not too close to the AUT, probe effects might be sufficiently small and might even be neglected (Hindman and Fooshe, 1998). Therefore the presented phaseless spherical algorithm neglects the probe correction for simplicity. Nevertheless it is possible to adapt the technique to one of the several probe corrected spherical near-field algorithms, e.g. Hansen (1988); Laitinen et al. (2005); Laitinen and Breinbjerg (2008); Leibfritz and Landstorfer (2006).

\section{Phaseless spherical algorithm}

The phaseless spherical algorithm determines a modal expansion of the AUT fields from amplitude only near-field measurements on two spheres. The individual steps are shown in the flowchart in Fig. 1. The algorithm starts with an initial guess for the mode expansion coefficients $\left\{Q^{\mathrm{IG}}\right\}$. The initial guess is important for the convergence of the algorithm and different choices are introduced and presented for the near-field scenarios in Sect. 4. Amplitude and phase nearfield values $\left\{E_{1}^{\mathrm{p}}\right\}$ are computed from the initial guess $\left\{Q^{\mathrm{IG}}\right\}$ according to Eq. (1). The propagated phase information on 


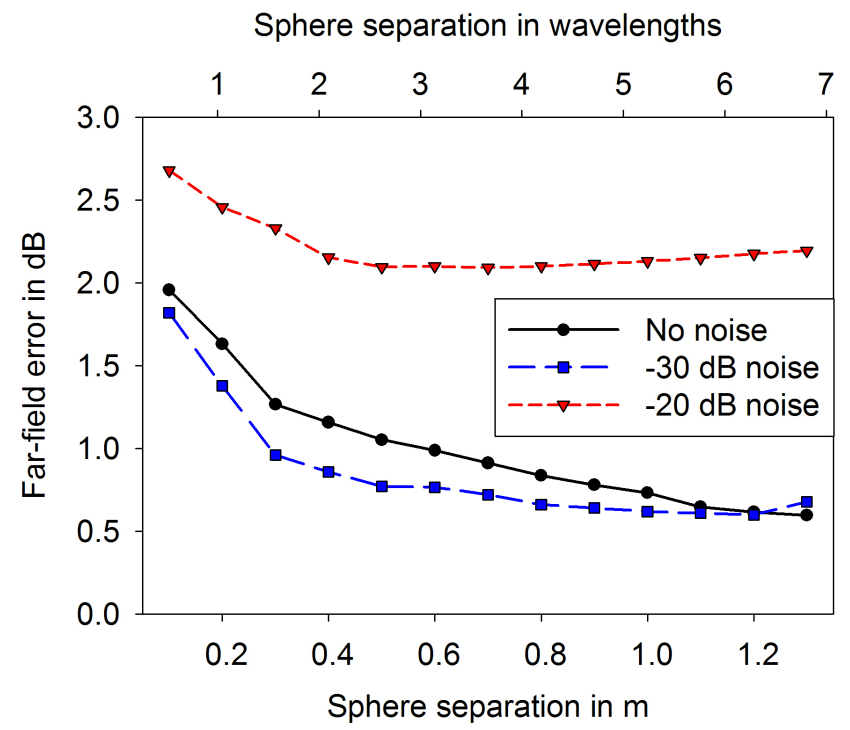

Fig. 2. Deviation of far-field pattern obtained by phaseless spherical algorithm from MoM reference pattern, unweighted average of all points on the sphere (step size $1^{\circ}$ in $\vartheta$ and $\varphi$ ). Near-field data with and without noise considered.

the first sphere is retained while the field amplitude is replaced by the measured amplitude $\left\{E_{1}^{\mathrm{m}}\right\}$ resulting in $\left\{E_{1}^{\mathrm{mp}}\right\}$. A new set of mode coefficients $\{Q\}$ is computed from $\left\{E_{1}^{\mathrm{mp}}\right\}$ according to Eq. (3). The same procedure is repeated for the second measurement sphere. During the iterations the error norm

$$
\epsilon=\frac{\sum_{i=1}^{S}\left\{\left(\sqrt{\left|E_{\varphi, i}^{\mathrm{p}}\right|^{2}+\left|E_{\vartheta, i}^{\mathrm{p}}\right|^{2}}-\sqrt{\left|E_{\varphi, i}^{\mathrm{m}}\right|^{2}+\left|E_{\vartheta, i}^{\mathrm{m}}\right|^{2}}\right)^{2}\right\}}{\sum_{i=1}^{S}\left\{\left|E_{\varphi, i}^{\mathrm{m}}\right|^{2}+\left|E_{\vartheta, i}^{\mathrm{m}}\right|^{2}\right\}}
$$

of the total field amplitude on the first measurement sphere is monitored as convergence metric. The subscripts $\varphi$ und $\vartheta$ denote the two polarisation components of the measured near-fields and $S$ is the total number of near-field samples on the sphere.

The mode expansion coefficients of an AUT are known to decrease rapidly. Filtering the higher order mode expansion coefficients and setting them to zero during the iterations helps the algorithm to find a modal expansion of the AUT, which incorporates this behaviour. So undesired rapid oscillations in the far-field pattern can be reduced. An appropriate filter index $i_{\text {filter }}$ is obtained by a hybrid preprocessing method (Razavi and Rahmat-Samii, 2006) which evaluates the main algorithm for a few iterations and various filter indices. $i_{\text {filter }}$ is chosen to minimise the error norm $\epsilon$.

An important parameter of the phaseless spherical algorithm is the separation of the measurement spheres. The lack of phase information in the transformation is overcomed by

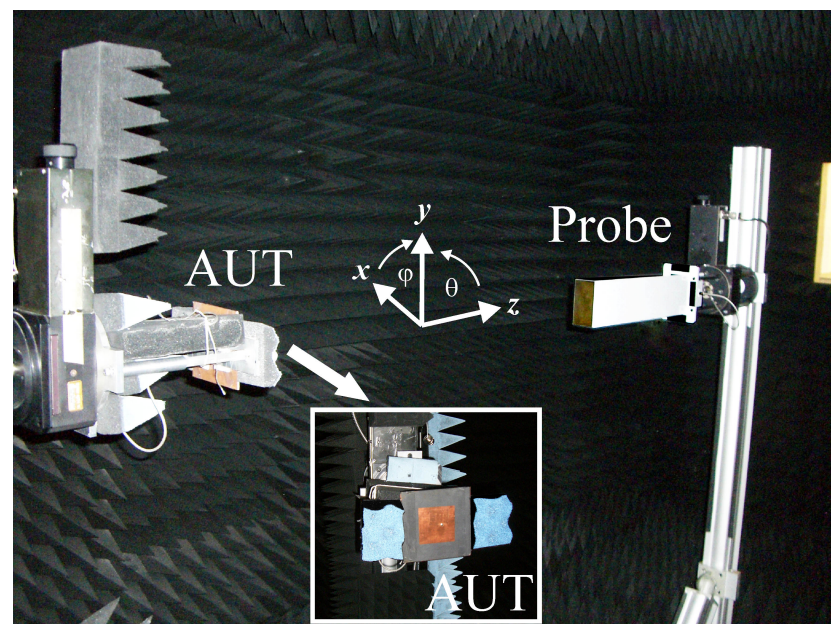

Fig. 3. Measurement setup inside the spherical near-field chamber at the Electrical Engineering Department, UCLA, with patch antenna as AUT measured at $1.57 \mathrm{GHz}$ and open ended waveguide probe.

additional amplitude information. Therefore it is required, that the measured amplitude pattern on the second sphere contains additional field information. This can be achieved by placing the first measurement sphere close to the AUT and the second sphere with a preferable high separation to the first one. To evaluate the minimum required sphere separation, a patch antenna (as shown in Sect. 4.1) is considered and electric field values are simulated for various sphere separations using the field simulator FEKO (http://www.feko.info). For the phaseless spherical algorithm, the first sphere remains constant whereas the radius of the second sphere is further increased. Figure 2 shows the deviation from the method of moments (MoM) reference far-field pattern, averaged over all points in the pattern. Also transformations have been carried out with additive noise added to the amplitude only near-field data. A high sphere separation is preferable in order to gain further field information on the second sphere with increasing measurement distance. According to this simplified analysis, a minimum sphere separation of at least two wavelengths is recommended.

\section{Results}

\subsection{Patch antenna measurement}

In the first example, the phaseless spherical algorithm is tested for a low gain patch antenna operating at $1.57 \mathrm{GHz}$. The measurement setup with positioner, AUT and open ended waveguide probe is shown in Fig. 3. The field distribution has been measured on two spheres of radii $1.7 \mathrm{~m}$ and $2.2 \mathrm{~m}$ employing a WR430 open-ended waveguide probe and a spherical NSI (Nearfield Systems Inc.) near-field scanner at UCLA. The initial guess $\left\{Q^{\mathrm{IG}}\right\}$ is obtained according 

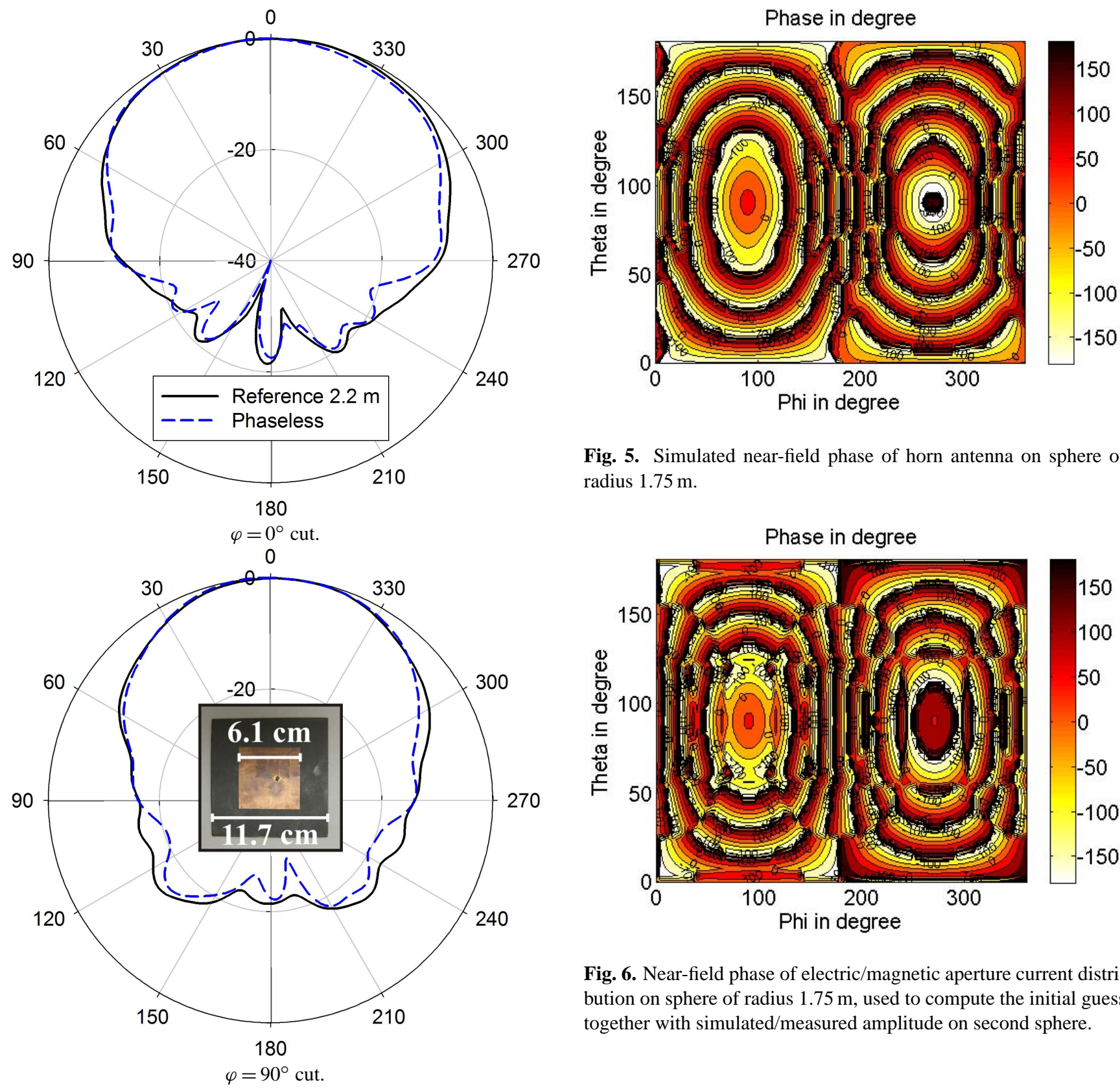

Fig. 5. Simulated near-field phase of horn antenna on sphere of radius $1.75 \mathrm{~m}$.

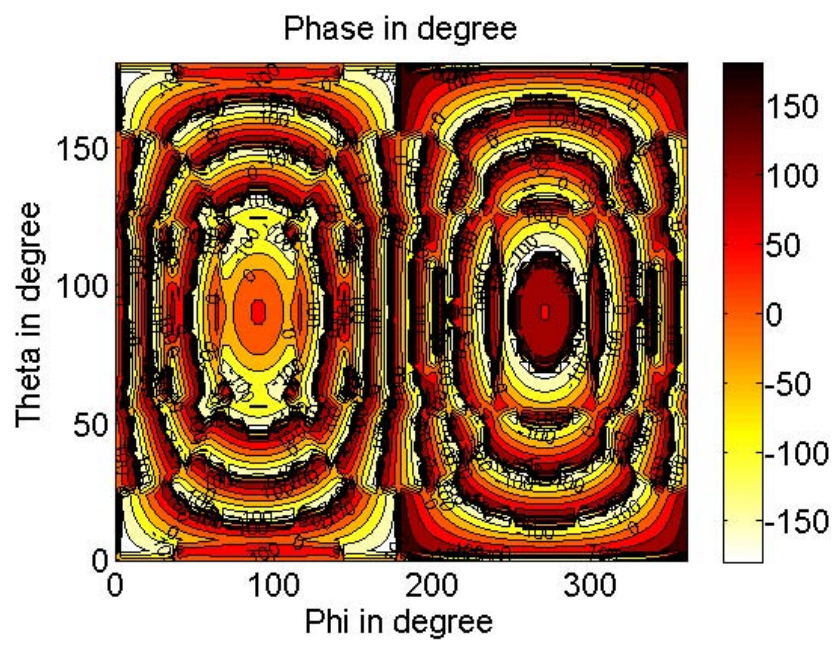

Fig. 6. Near-field phase of electric/magnetic aperture current distribution on sphere of radius $1.75 \mathrm{~m}$, used to compute the initial guess together with simulated/measured amplitude on second sphere.

Fig. 4. Comparison of patch antenna far-field patterns obtained by phaseless spherical algorithm from amplitude only data on two spheres with NSI reference pattern obtained from amplitude and phase data on a single sphere.

to Eq. (3) for the amplitude only data $\left\{E_{2}^{\mathrm{m}}\right\}$ measured on the second sphere. In order to have a reference, amplitude and phase have been measured and the far-field pattern has been computed with the NSI2000 software. Figure 4 shows the far-field pattern obtained by the phaseless spherical algorithm together with the NSI reference in the principal cuts. The patterns show an acceptable agreement to the reference if one keeps in mind that accurate amplitude and phase mea-

surements usually give more accurate results due to the complete description of the fields also by phase information.

During the measurement, the AUT positioner moves between AUT and probe while measuring the radiation in backward direction. This should be considered when evaluating measured patterns in backward direction of the AUT.

\subsection{Horn antenna simulation}

The second example considers a medium gain horn antenna at $6 \mathrm{GHz}$. A Narda standard gain horn model 642 is therefore simulated with FEKO. Near-field values have been computed on spheres of $1.27 \mathrm{~m}$ and $1.75 \mathrm{~m}$ radii. For the horn antenna two different initial guesses $\left\{Q^{\mathrm{IG}}\right\}$ are considered. The first one follows the same procedure as in the previous example. 

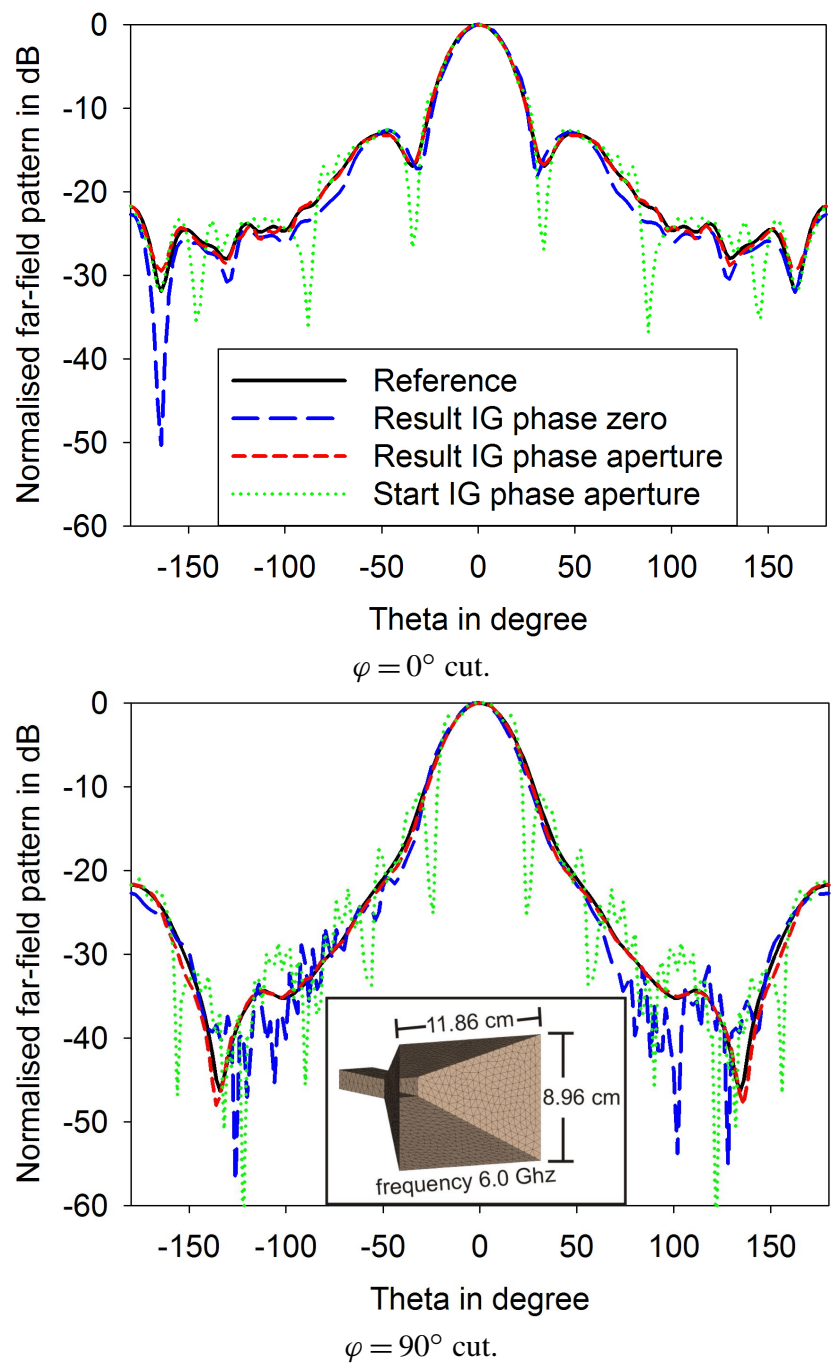

Fig. 7. Comparison of horn antenna far-field patterns obtained by phaseless spherical algorithm from amplitude only data on two spheres with MoM reference pattern.

The initial guess for the mode expansion coefficients is obtained from the amplitude only data on the second sphere (referred to as "result IG phase zero" in the figures). The second initial guess computes the field distribution of an electric/magnetic current distribution in the aperture of the horn. A uniform amplitude and phase distribution is assumed for the currents. The obtained phase on the second measurement sphere together with the simulated/measured amplitude is used to compute the initial guess (referred to as "result IG phase aperture" in the figures). Figure 5 shows the phase of the horn antenna on the second measurement sphere, whereas Fig. 6 shows the phase of the aperture current distribution. The aperture phase shows already close agreement with the real phase and so it is suited to compute the initial guess. The far-field pattern obtained by the phaseless spherical algorithm is shown in Fig. 7 in the principal cuts. The pattern

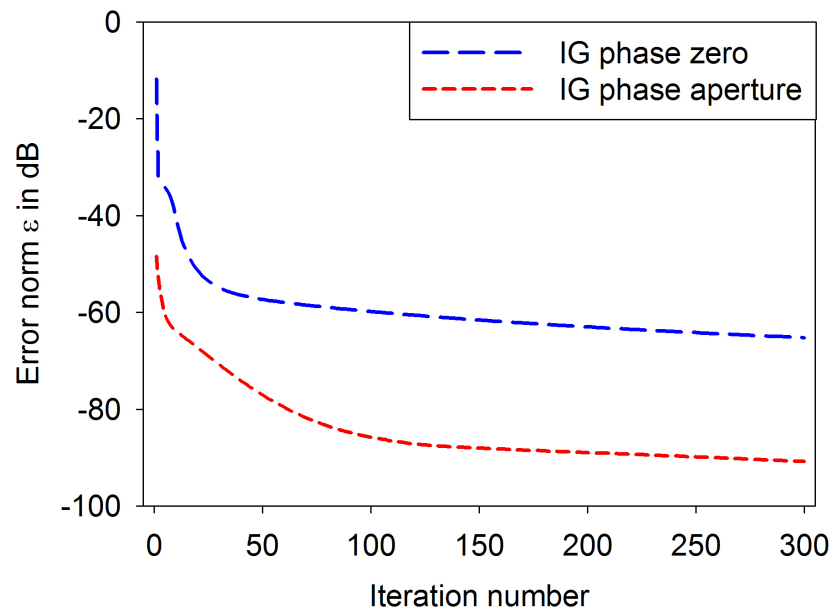

Fig. 8. Error norm $\epsilon$ of field amplitude error on first measurement sphere for phaseless spherical algorithm utilising simulated amplitude only near-field values on two spheres.

obtained by the second initial guess shows a much better performance than the first one. The green dotted curve shows the far-field pattern computed from the initial guess $\left\{Q^{\mathrm{IG}}\right\}$ employing the aperture phase distribution and demonstrates, how the far-field pattern is finally retrieved from the initial guess by the phaseless spherical algorithm resulting in the red short dashed curve. The error norm $\epsilon$ is plotted in Fig. 8 and shows a better performance for the initial guess employing the aperture phase distribution. This was expected since more a priori information of the AUT, in form of a simplified but good approximation of the phase distribution, was used to generate the initial guess.

\section{Conclusions}

A phaseless spherical near-field transformation algorithm has been presented. The algorithm determines the far-field pattern of an antenna from two amplitude only measurements on spheres of different radii in an iterative manner. Due to the spherical measurement configuration, the algorithm is not restricted to a particular class of antennas. Two different initial guesses for the mode expansion coefficients as starting point for the algorithm have been considered and evaluated. Simulated and measured results of a low gain patch antenna as well as a medium gain horn antenna have been presented, showing the applicability of the phaseless spherical algorithm for these types of antennas.

Acknowledgements. The authors are grateful to Shenheng $\mathrm{Xu}$, Electrical Engineering Department UCLA, for fruitful discussions.

This work was supported in part by a Ph.D. research fellowship of the German Academic Exchange Service (DAAD). 


\section{References}

Balanis, C. A.: Modern Antenna Handbook, John Wiley \& Sons, Inc., 2008

Costanzo, S., Massa, G. D., and Migliore, M. D.: A Novel Hybrid Approach for Far-Field Characterization From Near-Field Amplitude-Only Measurements on Arbitrary Scanning Surfaces, IEEE Trans. Antennas Propag., 53(6), 1866-1874, 2005.

FEKO Suite 5.5, http://www.feko.info, access: 2010.

Hansen, J. E.: Spherical Near-Field Antenna Measurements, IEE Electromagnetic Wave Series 26, Exeter, UK, 1988.

Hindman, G. and Fooshe, D. S.: Probe Correction Effects on Planar, Cylindrical and Spherical Near-Field Measurements, Antenna Measurement Techniques Association Conference, 1998.

Kerns, D. M.: Plane-Wave Scattering-Matrix Theory of Antennas and Antenna-Antenna Interactions, National Bureau of Standards, Boulder CO, 1981.

Laitinen, T. and Breinbjerg, O.: A First/Third-Order Probe Correction Technique for Spherical Near-Field Antenna Measurements Using Three Probe Orientations, IEEE Trans. Antennas Propag., 56(5), 1259-1268, 2008.

Laitinen, T., Pivnenko, S., and Breinbjerg, O.: Iterative Probe Correction Technique for Spherical Near-Field Antenna Measurements, IEEE Antennas Wireless Propag. Lett., 4, 221-223, 2005.

Las-Heras, F. and Sarkar, T.: A Direct Optimization Approach for Source Reconstruction and NF-FF Transformation Using Amplitude-Only Data, IEEE Trans. Antennas Propag., 50(4), 500-510, 2002
Leibfritz, M. and Landstorfer, F.: Full Probe Correction for NearField Antenna Measurements, Proc. IEEE AP-S Int. Symp., Albuquerque, NM, 2006.

Nearfield Systems Inc.: http://www.nearfield.com, access: 2010.

Pierri, R., D'Elia, G., and Soldovieri, F.: A Two Probes Scanning Phaseless Near-Field Far-Field Transformation Technique, IEEE Trans. Antennas Propag., 47(5), 792-802, 1999.

Razavi, S. F. and Rahmat-Samii, Y.: Phaseless Planar Near Field Measurements for Scanned Beams: Difficulties, a Hybrid Solution and Measured Results, Proc. IEEE AP-S Int. Symp., Albuquerque, NM, 2006.

Razavi, S. F. and Rahmat-Samii, Y.: On The Robustness Of Planar Phaseless Near-Field Measurements To Probe Positioning Errors, AMTA, Boston, MA, 2008.

Schejball, V., Kovarik, V., and Cermak, D.: Synthesized-ReferenceWave Holography for Determining Antenna Radiation Characteristics, IEEE Antenn. Propag. M., 50(5), 71-83, 2008.

Schmidt, C. H. and Rahmat-Samii, Y.: Phaseless Spherical NearField Antenna Measurements: Concept, Algorithm and Simulation, Proc. IEEE AP-S Int. Symp., Charleston, SC, 2009.

Soldovieri, F., Liseno, A., D'Elia, G., and Pierri, R.: Global Convergence of Phase Retrieval by Quadratic Approach, IEEE Trans. Antennas Propag., 53(10), 3135-3141, 2005.

Yaccarino, R. and Rahmat-Samii, Y.: Phaseless Bi-Polar Planar Near-Field Measurements and Diagnostics of Array Antennas, IEEE Trans. Antennas Propag., 47(3), 574-583, 1999.

Yaghjian, A. D.: An Overview of Near-Field Antenna Measurements, IEEE Trans. Antennas Propag., 34(1), 30-45, 1986. 